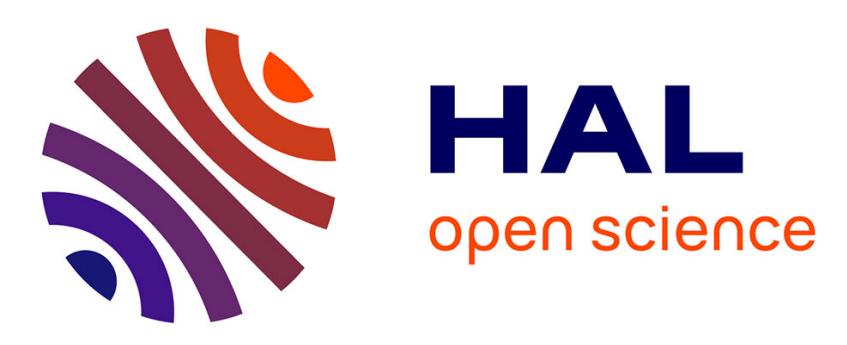

\title{
Effect of the laminar separation bubble induced transition on the hydrodynamic performance of a hydrofoil
}

\author{
Pierre Luc Delafin, François Deniset, Jacques-André Astolfi
}

\section{To cite this version:}

Pierre Luc Delafin, François Deniset, Jacques-André Astolfi. Effect of the laminar separation bubble induced transition on the hydrodynamic performance of a hydrofoil. European Journal of Mechanics - B/Fluids, 2014, Vol. 46, pp.190-200. 10.1016/j.euromechflu.2014.03.013 . hal-01087843

\section{HAL Id: hal-01087843 https://hal.science/hal-01087843}

Submitted on 26 Nov 2014

HAL is a multi-disciplinary open access archive for the deposit and dissemination of scientific research documents, whether they are published or not. The documents may come from teaching and research institutions in France or abroad, or from public or private research centers.
L'archive ouverte pluridisciplinaire $\mathbf{H A L}$, est destinée au dépôt et à la diffusion de documents scientifiques de niveau recherche, publiés ou non, émanant des établissements d'enseignement et de recherche français ou étrangers, des laboratoires publics ou privés. 


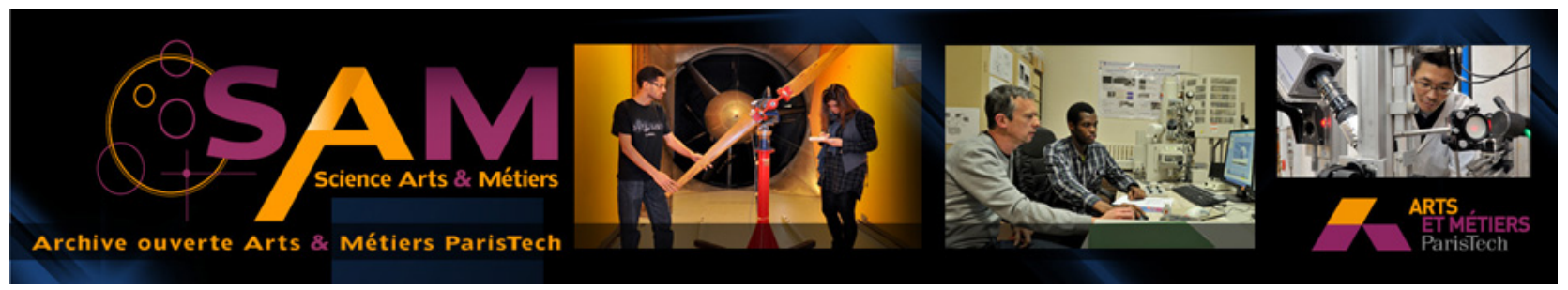

\section{Science Arts \& Métiers (SAM)}

is an open access repository that collects the work of Arts et Métiers ParisTech researchers and makes it freely available over the web where possible.

This is an author-deposited version published in: http://sam.ensam.eu

Handle ID: .http://hdl.handle.net/10985/8996

\section{To cite this version :}

Pierre Luc DELAFIN, François DENISET, Jacques-André ASTOLFI - Effect of the laminar separation bubble induced transition on the hydrodynamic performance of a hydrofoil - European Journal of Mechanics - B/Fluids - Vol. Vol. 46, p.190-200 - 2014 


\title{
Effect of the laminar separation bubble induced transition on the hydrodynamic performance of a hydrofoil
}

\author{
P.L. Delafin*, F. Deniset, J.A. Astolfi \\ Institut de Recherche de l'Ecole Navale EA 3634, BCRM Brest, Ecole Navale, CC 600, 29240 Brest, France
}

Keywords:

Lifting bodies

Laminar to turbulent transition

Laminar separation bubble

$\gamma-\operatorname{Re}_{\theta}$ transition model

\begin{abstract}
A B S T R A C T
The present study deals with the effect of the laminar separation bubble (LSB) induced transition on the lift, drag and moment coefficients of a hydrofoil. A 2D numerical study, based on the SST $\gamma-\operatorname{Re}_{\theta}$ transition model of ANSYS-CFX ${ }^{\circledR}$, is conducted on a NACA66 hydrofoil. Angles of attack range from $-4^{\circ}$ to $14^{\circ}$ and the chord-based Reynolds number is Re $=7.5 \times 10^{5}$. An experimental investigation is carried out in the French naval academy research institute's hydrodynamic tunnel based on the measurements of lift, drag and moment. Experiments on a smooth, mirror finished, hydrofoil enable comparison with RANS calculations using the transition model. Experiments with a roughness added on the leading edge enable comparison with RANS calculations using the SST fully turbulent model. For angles of attack below $6^{\circ}$, the LSB triggered laminar to turbulent transition of the boundary layers of the suction and pressure sides is located near the trailing edge of the smooth NACA66. As the angle of attack reaches $6^{\circ}$, the LSB suddenly moves to the leading edge on the suction side while transition is located at the trailing edge on the pressure side. The smooth hydrofoil shows higher $C_{\mathrm{L}}$ and $C_{\mathrm{M}}$ and lower $C_{\mathrm{D}}$ than the rough leading edge one from $-4^{\circ}$ to $6^{\circ}$. Both experiments lead to the same coefficients from $6^{\circ}$ to $14^{\circ}$. The calculations show that both models are in good agreement with their corresponding experiments. Velocity profiles in the vicinity of the LSB at an angle of attack of $2^{\circ}$ and pressure coefficients of the calculations using the transition model are compared with published experimental studies and show very good agreement. The SST $\gamma-\operatorname{Re}_{\theta}$ transition model proves to be a relevant, even essential, prediction tool for lifting bodies operating at a moderate Reynolds number.
\end{abstract}

\section{Introduction}

The need for practical RANS-based CFD codes including accurate laminar to turbulent transition models has increased due to the renewed interest in the low to moderate Reynolds number flows. The large increase of investigations in micro-aerodynamics and in micro-hydrodynamics dedicated to drones highlights the important need of valuable transition models at full scale. Performance prediction quality of flight control devices of micro-vehicles such as Autonomous Underwater Vehicles (AUVs), Unmanned Surface Vessels (USVs) or miniature Unmanned Air Vehicles (UAVs) strongly depends on the code capability to accurately model transition. Consideration of transition at model scale is also important to estimate performance of devices based on lifting bodies such as ship appendages (rudders, stabilizers, propulsion systems) or marine current turbines.

\footnotetext{
* Corresponding author. Tel.: +33 298233982.

E-mail address: pierre-luc.delafin@ecole-navale.fr (P.L. Delafin).
}

CFD investigations need to interact with theoretical and experimental approaches to allow accurate prediction of instantaneous forces on lifting bodies. These forces are very sensitive to flow separation, laminar separation bubble (LSB) and flow reattachment which are strongly influenced by boundary layer laminar to turbulent transition. The latter has been studied theoretically and experimentally for a long time in fluid dynamics. Its modeling is a very challenging task. Data analysis of classical flat plate cases with or without pressure gradient - and foil cases has given rise to dedicated transition models based on empirical correlations [1]. However, the absence of laminar to turbulent transition models in the Reynolds-Averaged Navier-Stokes (RANS) CFD codes has been considered as one of their major deficiencies for a long time. CFD calculations were then performed either in laminar regime or in fully turbulent regime. Empirical methods imposing a turbulence model downstream of a transition region or low-Reynolds-number models calibrated taking into account the transition prediction [2] were sometimes used but with no entire satisfaction.

Recently, transition models based on additional transport equations coupled to linear eddy-viscosity turbulent models have been proposed in the literature [3] and implemented in RANS-based CFD 


\begin{tabular}{|c|c|}
\hline \multicolumn{2}{|c|}{ Nomenclature } \\
\hline$\delta_{0.99}$ & thickness of the boundary layer \\
\hline$\gamma$ & intermittency \\
\hline$v$ & kinematic viscosity \\
\hline$v_{t}$ & turbulent viscosity \\
\hline$\rho$ & density \\
\hline AoA & Angle of Attack \\
\hline$b$ & hydrofoil span \\
\hline$c$ & hydrofoil chord length \\
\hline$C_{\mathrm{D}}$ & drag coefficient \\
\hline$C_{\mathrm{L}}$ & lift coefficient \\
\hline$C_{\mathrm{M}}$ & moment coefficient \\
\hline$C_{\mathrm{p}}$ & pressure coefficient \\
\hline$C_{\mathrm{f}}$ & friction coefficient \\
\hline LSB & Laminar separation bubble \\
\hline RANS & Reynolds-averaged Navier-Stokes \\
\hline $\operatorname{Re}$ & chord based Reynolds number $\left(=U_{\infty} * c / v\right)$ \\
\hline $\operatorname{Re}_{v}$ & strain-rate Reynolds number $\left(=y^{2} S / v\right)$ \\
\hline $\operatorname{Re}_{\theta}$ & momentum thickness Reynolds number $\left(=U_{\infty} \theta / \nu\right)$ \\
\hline SST & $\begin{array}{l}\text { Shear Stress Transport turbulence model based on } \\
\mathrm{k}-\omega \text { model }\end{array}$ \\
\hline SST-TM & SST coupled with $\gamma-\operatorname{Re}_{\theta}$ Transition Model \\
\hline$U_{\text {ext }}$ & velocity at $y=\delta_{0.99}$ \\
\hline$U_{\infty}$ & free stream velocity \\
\hline$U_{\tau}$ & friction velocity \\
\hline$y^{+}$ & dimensionless wall distance $\left(=y U_{\tau} / \nu\right)$ \\
\hline
\end{tabular}

codes, clearly improving their capabilities as compared to classical fully-turbulent models. This is the case of the correlation-based $\gamma-\operatorname{Re}_{\theta}$ transition model of Menter [4] validated by the help of flat plates and some industrial test cases. The model is coupled with the Shear Stress Transport (SST) k- $\omega$ turbulence model. Counsil and Goni Boulama propose a validation of Menter's model [5] based on the NACA 0012 airfoil for three Reynolds numbers $\left(5 \times 10^{4}, 1 \times 10^{5}\right.$ and $\left.2.5 \times 10^{5}\right)$ and three angles of attack $\left(0^{\circ}, 4^{\circ}\right.$ and $\left.8^{\circ}\right)$. Their results show good agreement between calculations with the transition model and experiments for instantaneous and mean flow features. Ducoin et al. [6] use this model on a NACA66 hydrofoil undergoing transient pitching motions. Numerical and experimental pressure coefficients are compared at several chord locations for both quasi-static and high angular velocities and show good agreement. The inflection point of the $C_{\mathrm{p}}$ curves due to transition is particularly well predicted by the transition model. Lanzafame et al. [7], after having validated Menter's model on a 2D S809 airfoil, use it with success on a horizontal-axis wind turbine. A validation of the $\mathrm{k}-\mathrm{k}_{\mathrm{l}}-\omega$ model of Walters and Cokljat [8] based on one additional transport equation is carried out by Genç, Kaynak and Yapici [9]. They compare the $\mathrm{k}-\mathrm{k}_{l}-\omega$ model to Menter's model on a NACA 2415 airfoil at AoA $=8^{\circ}$ and $\operatorname{Re}=2 \times 10^{5}$. Their results show that modeling the transition improves the accuracy of the solution for a moderate Reynolds number compared to a fully turbulent calculation. The $\mathrm{k}-\mathrm{k}_{l}-\omega$ model seems to be more accurate on the studied configuration.

High accuracy requirements lead to the use of high-density grids. As a consequence, High Performance Computing (HPC) capabilities are required to use single-point eddy viscosity models instead of correlation-based highly empirical approaches only. Conversely, due to another limitation consisting in reasonable calculation times, these models are less time-consuming as compared to fully-realized methods (LES, DNS) which cannot be easily considered on a day-to-day basis for classical engineering applications.

The purpose of this study is to evaluate the capability of the $\gamma-\operatorname{Re}_{\theta}$ Menter two-equation transition model [4] in accurately determining flow characteristics on a NACA 66(mod)-312 hydrofoil in incompressible flow for a moderate Reynolds number $(\operatorname{Re}=$

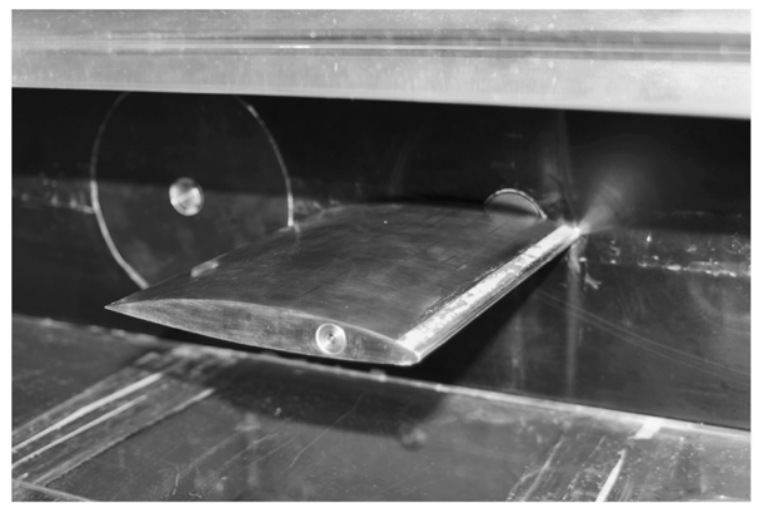

Fig. 1. NACA 66(mod)-312 in the test section, with leading edge roughness.

$7.5 \times 10^{5}$ ) at angles of attack ranging from $-4^{\circ}$ to $14^{\circ}$. The model is based on two additional transport equations, one dedicated to the intermittency $\gamma$ and the other one to the Reynolds number $\operatorname{Re}_{\theta}$ based on boundary layer displacement thickness. First, the experimental setup is presented, followed by a description of the model and an overview of the numerical methodology. Then, after a verification procedure dealing with four spatial and four temporal discretizations, results are presented and discussed. A global analysis dealing with lift, drag and moment coefficients is followed by a local analysis based on velocity profiles in the vicinity of the LSB, and on pressure and friction coefficients. Special focus is carried out in the boundary layer on transition location. 3D calculations are run to evaluate the 3D effect inherent in the experiment and complete the $2 \mathrm{D}$ calculations. The calculation accuracy improvement due to the consideration of transition effect is highlighted on the basis of validations with measurements carried out at the French naval academy research institute (IRENav).

\section{Experimental study}

The calculations presented in this paper are validated by experiments carried out in the IRENav's cavitation tunnel. The dimensions of the test section are $1 \mathrm{~m}$ (length) $\times 0.192 \mathrm{~m}$ (height) $\times 0.192 \mathrm{~m}$ (width). The $150 \mathrm{~mm}$ chord, mirror finished, NACA 66 (mod)-312 spanned the entire width of the test section (Fig. 1). Experiments were carried out at a flow velocity $U_{\infty}=5 \mathrm{~m} \mathrm{~s}^{-1}$ which corresponds to $\operatorname{Re}=7.5 \times 10^{5}$. Atmospheric pressure was set in the test section and no cavitation was observed. The inlet turbulence intensity was measured at 3\%. A 3-component hydrodynamic balance was used to measure lift, drag and moment. Each data acquisition was led during $10 \mathrm{~s}$ with a sample frequency of $1 \mathrm{kHz}$. Angles of attack ranged from $-4^{\circ}$ to $14^{\circ}$ with a general step of $1^{\circ}$ locally refined to $0.5^{\circ}$. The axis of rotation was located at $25 \%$ of the chord from the leading edge. Experiments have also been carried out on the same hydrofoil with an added roughness on the leading edge (Fig. 1) so that the laminar to turbulent transition was triggered at the leading edge. The added roughness consisted in layers of sandpaper (gritsize $=15 \mu \mathrm{m}$ ) covering the first $5 \%$ of both pressure and suction sides.

The accuracy of the balance was $\pm 0.4 \%$ for the lift, $\pm 0.4 \%$ for the drag and $\pm 0.7 \%$ for the moment. Fluctuations of the velocity control led to an average accuracy of the reference velocity $U_{\infty}$ of $\pm 0.05 \mathrm{~m} \mathrm{~s}^{-1}$.

\section{Model and numerical methods}

\subsection{Geometry and mesh}

The hydrofoil is a $150 \mathrm{~mm}$ chord NACA 66(mod)-312 as in the experiments. 2D and 3D computational domains have been considered in this study. The 2D computational domain has the height of 


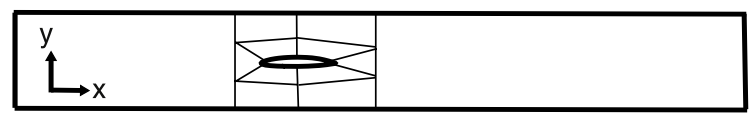

Fig. 2. Topology of the computational domain.

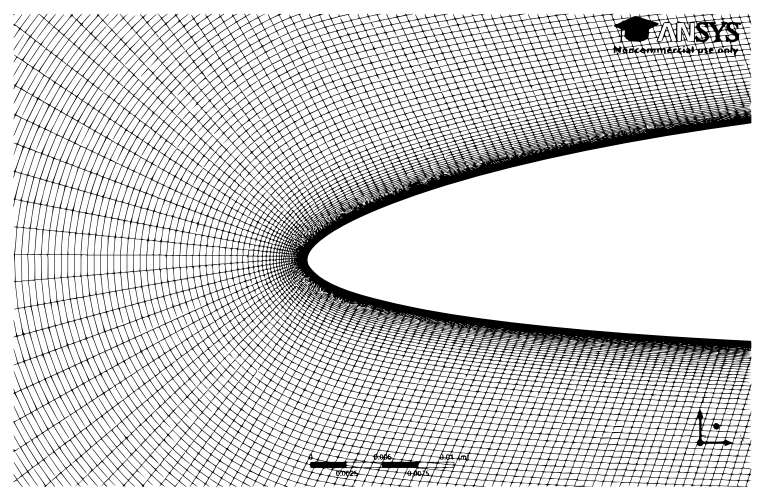

Fig. 3. High-density mesh close to the leading edge.

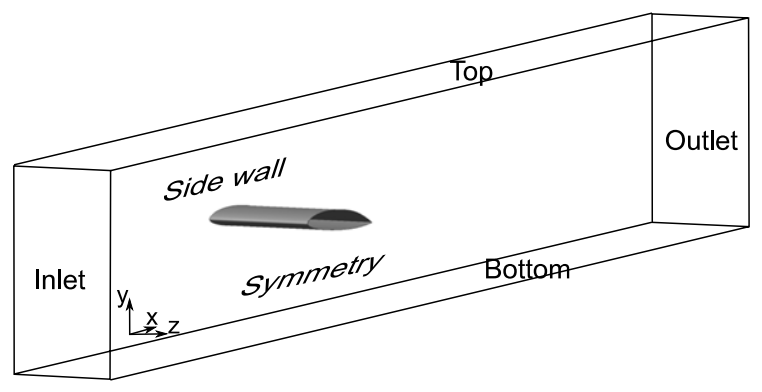

Fig. 4. 3D computational domain.

the test section and extends 3 chords upstream the hydrofoil and 6 chords downstream (Fig. 2). The $\mathrm{O}-4 \mathrm{H}$ grid is created and smoothed with ANSYS ICEM-CFD [10]. The foil is discretized by 742 nodes (Fig. 3) and the domain contains 160,000 hexahedral elements. The mesh is voluntarily dense to accurately capture transition and detachment. Maximum $y^{+}$value is kept of the order of 1 during simulations and the grid expansion ratios never exceed 1.2 close to the foil.

The 3D computational domain (Fig. 4) represents half the span of the experimental test section. A symmetry condition is imposed on the mid span face. The grid is based on a $2 \mathrm{D}$ grid containing 50,000 hexaedral elements and the foil is discretized by 290 nodes. $y^{+}$is kept close to one on the foil $\left(y_{\max }^{+}=3.46\right.$ and $y_{\text {averaged }}^{+}=1.0$ ). Top and bottom walls are meshed with large $y^{+}$adapted to wall functions while the wall on which the hydrofoil is fixed (side wall) is meshed with $y^{+}$values close to $1\left(y_{\max }^{+}=4.6\right.$ and $y_{\text {averaged }}^{+}=$ 1.0 ). The fine resolution of the side wall intends to capture accurately the horseshoe vortex that develops at the junction between the foil and the wall [11] and induces 3D effects. Fig. 5 shows a close view of the grid near the foil-wall junction. The 2D mesh is extruded by 64 layers with a ratio of 1.13 so that the final mesh contains $3.3 \times 10^{6}$ hexaedral cells. Inlet and outlet boundary conditions are the same as in 2D simulations.

\subsection{Model}

The physical model is based on the mass and momentum conservation equations. The fluid is considered viscous and incompressible. The k- $\omega$ SST (Shear Stress Transport) closure turbulence model [12] is used since it is known to predict better boundary layers submitted to adverse pressure gradients than the other twoequation RANS turbulence models [13]. The SST model is compared

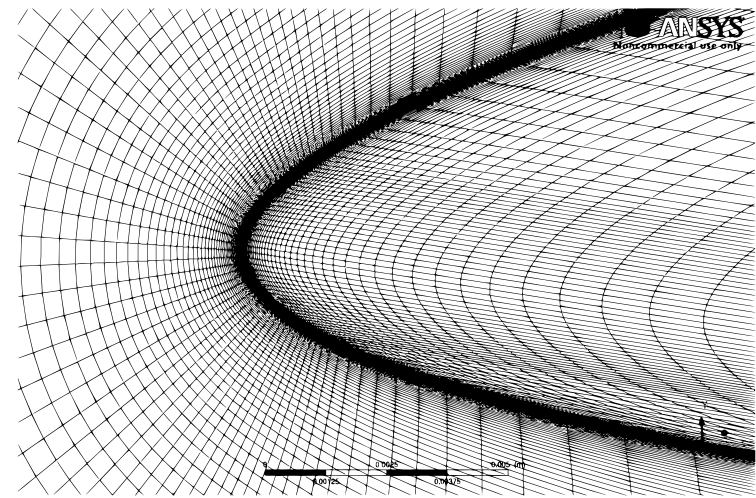

Fig. 5. High-density mesh close to the leading edge and the side wall.

with the two transport equations $\gamma-\operatorname{Re}_{\theta}$ transition model [4]. The transition model is coupled with the SST turbulence model by the turbulent kinetic energy transport equation (Eq. (1)). The production and destruction terms of $k\left(\tilde{P}_{k}\right.$ and $\left.\tilde{D}_{k}\right)$ are functions of the effective intermittency $\gamma_{\text {eff }}$ and the original terms of the SST model $\left(P_{k}\right.$ and $\left.D_{k}\right)$, as written in Eq. (3). The transport equation for the specific dissipation rate $\omega$ (Eq. (2)) is not affected by the coupling.

$$
\begin{aligned}
& \frac{\partial(\rho k)}{\partial t}+\frac{\partial\left(\rho U_{j} k\right)}{\partial x_{j}}=\tilde{P}_{k}-\tilde{D}_{k}+\frac{\partial}{\partial x_{j}}\left(\left(\mu+\sigma_{k} \mu_{t}\right) \frac{\partial k}{\partial x_{j}}\right) \\
& \frac{\partial(\rho \omega)}{\partial t}+\frac{\partial\left(\rho U_{j} \omega\right)}{\partial x_{j}}=\alpha \frac{P_{k}}{v_{t}}-D_{\omega}+C d_{\omega} \\
& +\frac{\partial}{\partial x_{j}}\left(\left(\mu+\sigma_{\omega} \mu_{t}\right) \frac{\partial \omega}{\partial x_{j}}\right)
\end{aligned}
$$

The first equation of the transition model is for the intermittency $\gamma$ (Eq. (5)) which is used to turn on the production term of the turbulent kinetic energy downstream of the transition point. The production term $P_{\gamma}$ is designed to be equal to zero in the laminar boundary layer and active where the transition criteria is reached. It is controlled by an onset function depending on the ratio of the momentum thickness Reynolds number $\operatorname{Re}_{\theta}$ and the critical Reynolds number $\operatorname{Re}_{\theta c}$. To avoid non-local operations, $\operatorname{Re}_{\theta}$ is related to the strain-rate Reynolds number $\mathrm{Re}_{v}$ by an empirical correlation (Eq. (4)).

$\operatorname{Re}_{\theta}=\frac{\max \left(\operatorname{Re}_{v}\right)}{2.193} ; \quad \operatorname{Re}_{v}=\frac{\rho y^{2} S}{\mu}$

$\operatorname{Re}_{v}$ only depends on density, wall distance, strain-rate and viscosity and can therefore be calculated on each grid node. $P_{\gamma}$ is limited so that the intermittency cannot exceed 1 . The destruction term $E_{\gamma}$ ensures that the intermittency keeps very low values in the boundary layer and enables the prediction of relaminarization when the transition criteria is no longer met. A correction was done to take into account the separation induced transition, allowing the local intermittency to exceed 1 when the boundary layer separates. This results in a large production of $k$ and in an earlier reattachment of the boundary layer, so that the transition length can be correctly predicted. The intermittency equation requires data obtained from correlations. These correlations relate $\operatorname{Re}_{\theta t}$ to the free stream turbulence intensity $\mathrm{Tu}$ and the pressure gradient $d p / d s$ and are then non-local. Eq. (6) is designed to transport the scalar $\overline{\operatorname{Re}}_{\theta t}$. The production term $P_{\theta t}$ ensures that $\overline{\operatorname{Re}}_{\theta t}$ matches the value of $\operatorname{Re}_{\theta t}$ calculated from the empirical correlation in the free stream. A blending function turns $P_{\theta t}$ off in the boundary layer so that $\overline{\operatorname{Re}}_{\theta t}$ is diffused in it from the free stream. Eq. (6) then takes 
non-local empirical correlations and transforms them into a local quantity so that the intermittency equation can be solved.

$$
\begin{aligned}
& \frac{\partial(\rho \gamma)}{\partial t}+\frac{\partial\left(\rho U_{j} \gamma\right)}{\partial x_{j}}=P_{\gamma}-E_{\gamma}+\frac{\partial}{\partial x_{j}}\left(\left(\mu+\frac{\mu_{t}}{\sigma_{f}}\right) \frac{\partial \gamma}{\partial x_{j}}\right) \\
& \frac{\partial\left(\rho \overline{\operatorname{Re}}_{\theta t}\right)}{\partial t}+\frac{\partial\left(\rho U_{j} \overline{\operatorname{Re}}_{\theta t}\right)}{\partial x_{j}}=P_{\theta t}+\frac{\partial}{\partial x_{j}}\left(\sigma_{\theta t}\left(\mu+\mu_{t}\right) \frac{\partial \overline{\operatorname{Re}}_{\theta t}}{\partial x_{j}}\right) .
\end{aligned}
$$

Here, the intermittency only controls the production of turbulent kinetic energy. It does not appear in the eddy viscosity definition. The empirical correlations allow this model to predict different transition mechanisms like natural transition, bypass transition or separation induced transition. Calculations carried out with the fully turbulent $k-\omega$ SST model only will be referred to as SST while those carried out with the $k-\omega$ SST model coupled with the $\gamma-\operatorname{Re}_{\theta}$ transition model will be referred to as SST-TM henceforth.

\subsection{Boundary conditions}

Calculations are carried out in water (density $\rho=997 \mathrm{~kg} \mathrm{~m}^{-3}$, kinematic viscosity $v=1 \times 10^{-6} \mathrm{~m}^{2} \mathrm{~s}^{-1}$ ). The inlet velocity is set to $U_{\infty}=5 \mathrm{~m} \mathrm{~s}^{-1}$ so that the chord based Reynolds number equals $7.5 \times 10^{5}(c=0.15 \mathrm{~m})$. Inlet turbulence intensity is set to $3 \%$ as in the experiments. An outlet condition with a 0 Pa relative static pressure is imposed on the downstream boundary. Top and bottom faces are set as symmetry to limit the number of cells. This is possible since the cavitation tunnel corresponding walls are slightly divergent to avoid the slight blockage due to the boundary layers' development on these faces. Though not presented in this paper, calculations with walls instead of symmetries have been run and showed very little difference when compared to the calculations with symmetries. Front and back faces are set as symmetry in the 2D calculation whereas in the 3D calculation, the side wall (Fig. 4) is set as wall and a symmetry condition is imposed on the mid span face. Lastly, a wall condition is imposed on the foil.

Calculations are run with both SST and SST-TM models for angles of attack between $\mathrm{AoA}=-4^{\circ}$ and $\mathrm{AoA}=14^{\circ}$ with a step of $1^{\circ}$ locally refined to $0.5^{\circ}$. The axis of rotation is located at the quarter of the chord, as in the experiments.

\subsection{Numerical method}

The problem is solved by the finite volumes method [14], using the CFD RANS based code CFX ${ }^{\circledR}$ [15]. Advection terms are calculated by a hybrid first/second order scheme High Resolution. A blend factor makes it switch from a first order scheme in regions of high spatial gradients to ensure robustness, to a second order scheme in regions of low gradients to ensure accuracy. The temporal discretization is achieved by using the implicit second order backward Euler scheme. Calculations are run in double precision and are parallelized on 4 Intel Q9550 $2.83 \mathrm{GHz}$ cores. Convergence criteria target value on residuals is set as $10^{-5}$.

\section{Verification}

Several SST-TM calculations have been run to assess the independence of the solution to the spatial and temporal discretizations and also to the residuals target. Requirements of the $\gamma-\operatorname{Re}_{\theta}$ transition model being higher than those of the fully turbulent model, convergence will only be studied with SST-TM calculations.

\subsection{Grid}

Calculations have been run at $\mathrm{AoA}=3^{\circ}$ and $\mathrm{AoA}=8^{\circ}$ with four different grids referenced in Table 1 . Three of these grids have
Table 1

Grid resolutions and $y_{\max }^{+}$values at $\mathrm{AoA}=8^{\circ} . N_{\text {foil }}$ is the number of nodes on the foil, $\operatorname{Re}=7.5 \times 10^{5}$.

\begin{tabular}{llll}
\hline Mesh & nb of cells & $\begin{array}{l}y_{\max }^{+} \\
\text {(foil) }\end{array}$ & $N_{\text {foil }}$ \\
\hline M1 & 160,000 & 1.3 & 742 \\
M2 & 102,000 & 1.1 & 424 \\
M3 & 68,000 & 1.2 & 242 \\
M4 & 31,500 & 2.6 & 230 \\
\hline
\end{tabular}

Table 2

\begin{tabular}{|c|c|c|c|c|}
\hline \multirow[t]{2}{*}{ Mesh } & \multicolumn{2}{|l|}{$3^{\circ}$} & \multicolumn{2}{|l|}{$8^{\circ}$} \\
\hline & $C_{\mathrm{L}}$ & $C_{\mathrm{D}}$ & $C_{\mathrm{L}}$ & $C_{\mathrm{D}}$ \\
\hline M1 & 0.649 & 0.0071 & 1.032 & 0.0210 \\
\hline M2 & $-0.59 \%$ & $+0.24 \%$ & $-0.51 \%$ & $+0.54 \%$ \\
\hline M3 & $-1.86 \%$ & $+1.26 \%$ & $-0.17 \%$ & $+0.18 \%$ \\
\hline M4 & $-2.78 \%$ & $+4.51 \%$ & $-0.57 \%$ & $+2.93 \%$ \\
\hline
\end{tabular}

Mesh convergence for $\mathrm{AoA}=3^{\circ}$ and $\mathrm{AoA}=8^{\circ}, \operatorname{Re}=7.5 \times 10^{5}$.

Table 3

CPU time requirements to reach convergence at $\mathrm{AoA}=3^{\circ}, \mathrm{Re}=7.5 \times 10^{5}$.

\begin{tabular}{lll}
\hline \multirow{2}{*}{ Mesh } & \multicolumn{2}{c}{ CPU time $\left(\times 10^{3} \mathrm{~s}\right)$} \\
\cline { 2 - 3 } & SST & SST-TM \\
\hline M1 & 4.66 & 24.1 \\
M2 & 3.44 & 15.5 \\
M3 & 2.16 & 12.8 \\
M4 & 0.916 & 4.99 \\
\hline
\end{tabular}

a $y_{\max }^{+}$close to 1 at $A o A=8^{\circ}$. Mesh M4 is a coarse mesh of 31,500 cells. M3 has the same number of cells on the hydrofoil as M4 but the direction normal to the wall in the O-mesh, as well as the upstream and downstream areas are refined so that the number of cells reaches 68,000 . M2 is built multiplying by 2 the number of cells on the pressure and suction sides compared to M3. The number of cells on both leading and trailing edges is increased from 40 to 50 . Other edges of the mesh (Fig. 2) have the same number of cells as M3. M1 is built multiplying by 2 the number of cells on the pressure and suction sides compared to M2. All the other edges of the mesh have the same number of cells as M2.

Lift and drag coefficients calculated on grid M1 are displayed in Table 2. $C_{\mathrm{L}}$ and $C_{\mathrm{D}}$ obtained on grids $\mathrm{M} 2, \mathrm{M} 3$ and $\mathrm{M} 4$ are expressed as the deviation (\%) from M1 coefficients. Grid M2 shows very little difference with grid M1 for both lift and drag coefficients at AoA $=3^{\circ}$ and stays below the demanded level of $1 \%$. Grids M3 and M4 however show increasing deviations. At AoA $=8^{\circ}, C_{\mathrm{L}}$ and $C_{D}$ of grids $M 2$ and $M 3$ are very close to those of grid M1. The surprising accuracy of grid M3 was unexpected. Grid M4 gives a good prediction of $C_{\mathrm{L}}$ but a slightly overestimated $C_{\mathrm{D}}$. According to these two cases, grid M2 can be considered spatially converged. However, the high density grid M1 will be used in this study to have a higher accuracy on the transition location.

Table 3 displays the CPU time required by calculations at $\mathrm{AoA}=$ $3^{\circ}$ to reach convergence on grids M1-M4 with both SST and SST-TM models. SST calculations prove to be about five times faster than SST-TM calculations. It should however be noted that SST calculations only require steady state simulations while SST-TM calculations require transient simulations.

\subsection{Time step}

SST calculations converge easily with a steady state simulation until an AoA of $13^{\circ}$. At $13.5^{\circ}$, the steady state simulation leads to oscillating $C_{\mathrm{L}}$ and $C_{\mathrm{D}}$ and it is necessary to switch to a transient simulation. However vortex shedding has not started and a steady state solution is reached. 
Table 4

Time step convergence at $\mathrm{AoA}=3^{\circ}$ and $\mathrm{AoA}=8^{\circ}, \operatorname{Re}=7.5 \times 10^{5}$.

\begin{tabular}{|c|c|c|c|c|}
\hline \multirow[t]{2}{*}{$\Delta t(\mathrm{~s})$} & \multicolumn{2}{|l|}{$3^{\circ}$} & \multicolumn{2}{|l|}{$8^{\circ}$} \\
\hline & $C_{\mathrm{L}}$ & $C_{\mathrm{D}}$ & $C_{\mathrm{L}}$ & $C_{D}$ \\
\hline $2.5 \times 10^{-4}$ & - & - & 1.032 & 0.0210 \\
\hline $5 \times 10^{-4}$ & 0.649 & 0.0071 & $+0.00 \%$ & $-0.01 \%$ \\
\hline $10^{-3}$ & $+0.006 \%$ & $+0.001 \%$ & $\pm 0.20 \%$ & $\pm 0.75 \%$ \\
\hline $5 \times 10^{-3}$ & $\pm 0.75 \%$ & $\pm 4.60 \%$ & $-0.70 \%$ & $+6.35 \%$ \\
\hline
\end{tabular}

Table 5

Results of the residual study ( $\mathrm{SS}=$ Suction side, $\mathrm{PS}=$ Pressure side), $\mathrm{AoA}=3^{\circ}$, $\operatorname{Re}=7.5 \times 10^{5}$.

\begin{tabular}{lllll}
\hline & $10^{-8}$ & $10^{-6}$ & $10^{-5}$ & $10^{-4}$ \\
\hline$C_{\mathrm{L}}$ & 0.649 & $+0 \%$ & $+0 \%$ & $-0.012 \%$ \\
$C_{\mathrm{D}}$ & 0.0071 & $+0 \%$ & $+0.23 \%$ & $-0.044 \%$ \\
$x / c$ transition SS & 0.8005 & 0.8004 & 0.8004 & 0.8004 \\
$x / c$ transition PS & 1 & 1 & 1 & 1 \\
\hline
\end{tabular}

Convergence of SST-TM calculations is more difficult and nearly always requires a transient simulation, even if a steady state solution is often reached. Three different time steps are tested on mesh M1 at $\mathrm{AoA}=3^{\circ}$ and $\mathrm{AoA}=8^{\circ}: 5 \times 10^{-4} \mathrm{~s}, 10^{-3} \mathrm{~s}$ and $5 \times 10^{-3} \mathrm{~s}$. An additional time step has been tested at $A o A=8^{\circ}: 2.5 \times 10^{-4} \mathrm{~s}$. The results are presented in Table 4.

At $\mathrm{AoA}=3^{\circ}$, results show very little difference between $\mathrm{d} t=$ $5 \times 10^{-4} \mathrm{~s}$ and $\mathrm{d} t=10^{-3} \mathrm{~s}(\ll 1 \%)$ while $\mathrm{d} t=5 \times 10^{-3} \mathrm{~s}$ shows oscillations of $C_{\mathrm{L}}$ and $C_{\mathrm{D}}$ with a significant amplitude (especially on $\left.C_{\mathrm{D}}\right) \cdot \mathrm{d} t=10^{-3} \mathrm{~s}$ is then adopted for low AoA calculations. At AoA $=$ $8^{\circ}$, results show very little difference between $\mathrm{d} t=2.5 \times 10^{-4} \mathrm{~s}$ and $\mathrm{d} t=5 \times 10^{-4} \mathrm{~s}(\ll 1 \%)$ while $\mathrm{d} t=10^{-3} \mathrm{~s}$ and $\mathrm{d} t=5 \times 10^{-3} \mathrm{~s}$ lead to oscillating results. $\mathrm{d} t=5 \times 10^{-4} \mathrm{~s}$ is then adopted for high AoA calculations.

\subsection{Residuals}

Calculations have been run with different levels of residual target to assess the independence of the solution to this parameter. SST-TM calculations are transient. Therefore, the residual target is reached within each physical time step. Results of the calculations are summarized in Table 5.

Table 5 shows that the residual levels of $10^{-8}, 10^{-6}, 10^{-5}$ and even $10^{-4}$ all lead to very similar results. A residual target of $10^{-5}$ is the highest level that leads to $0 \%$ deviation on the lift coefficient. The value $10^{-5}$ is chosen for the calculations.

\section{Results and discussion}

The laminar to turbulent transition mechanism that operates in this study is the separation-induced transition, also called laminar separation bubble (LSB) induced transition. This mechanism occurs when a laminar boundary layer is subjected to a sufficiently strong adverse pressure gradient that induces separation of the boundary layer. The Kelvin-Helmholtz instabilities are developed and amplified in the separated shear layer and finally lead to a breakdown of the shear layer, resulting in a turbulent flow that reattaches to the foil $[16,17]$. A closed bubble (the LSB) is formed between the separation and reattachment points. Considering the flow around a hydrofoil, the chordwise location of the LSB mainly depends on the pressure gradient, the Reynolds number, the free stream turbulence intensity and the surface roughness. The pressure gradient itself depends on the foil geometry and the angle of attack. The transition location then moves on both suction and pressure sides while varying the angle of attack. In the particular case of a NACA 6-series foil at a moderate Re, the suction side transition location moves suddenly from the trailing edge to the leading edge, as shown in Fig. 6. Laminar separation
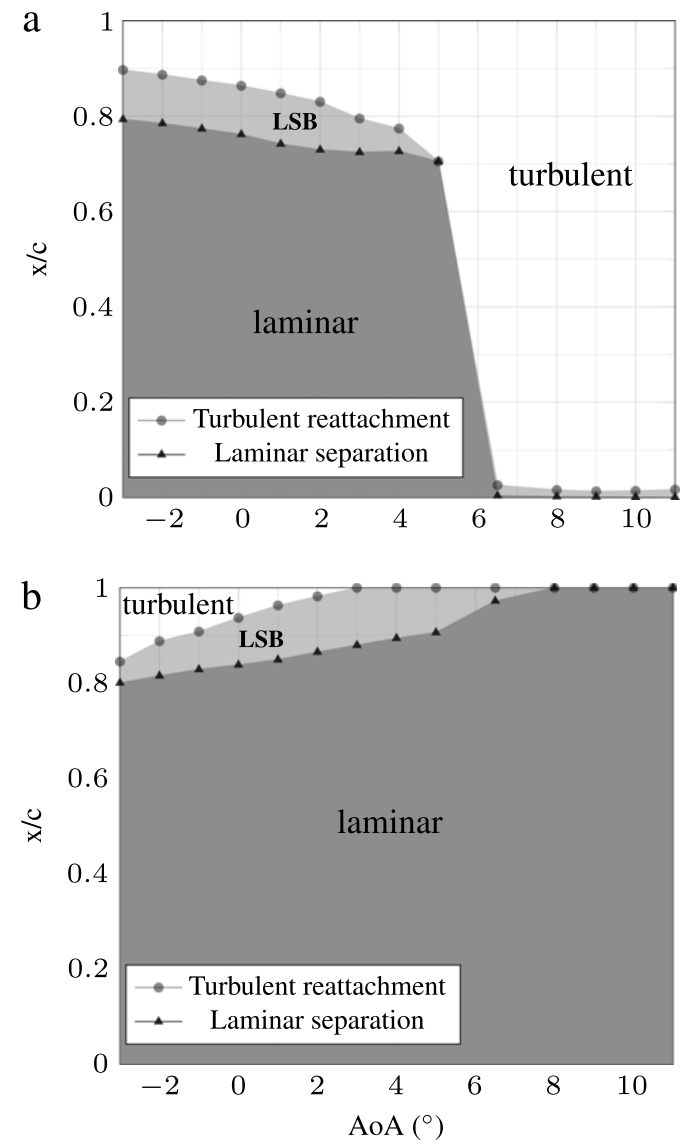

Fig. 6. Laminar separation and turbulent reattachment locations on suction side (a) and pressure side (b) as a function of the angle of attack, Re $=7.5 \times 10^{5}$.

and turbulent reattachment locations plotted in Fig. 6 are obtained from $C_{\mathrm{f}}$ extractions of 2D SST-TM calculations. The transition location is considered as the closure of the $\operatorname{LSB}\left(C_{\mathrm{f}}=0\right)$.

\subsection{Force predictions}

In this section, both SST and SST-TM models have been employed and compared to experiments in terms of lift, drag and moment coefficients.

\subsubsection{Lift coefficient}

Fig. 7(a) shows $C_{\mathrm{L}}$ for both smooth and rough leading edges experiments that are compared with SST-TM and SST calculations respectively. Smooth leading edge data show a $C_{\mathrm{L}}$ evolution in 3 parts : for $A o A \in\left[-4^{\circ} ; 5^{\circ}\right]$ evolution is quasi-linear with a slight increase of the slope for $\mathrm{AoA} \in\left[2^{\circ} ; 5^{\circ}\right]$. Then a plateau at $C_{\mathrm{L}}=0.8$ takes place from AoA $=5^{\circ}$ to $\mathrm{AoA}=6^{\circ} . C_{\mathrm{L}}$ increases again from AoA $=6^{\circ}$ to AoA $=14^{\circ}$ with a lower slope than in the first part. $C_{\mathrm{L}}$ starts oscillating at $\mathrm{AoA}=13^{\circ}$ due to vortex shedding but average values keep rising. The shape of this curve is specific to NACA 6-series foils operating at moderate Reynolds numbers. The lift plateau is generated by the motion of the suction side transition from the trailing edge to the leading edge combined with the shortening of the pressure side LSB at the trailing edge (Fig. 6). The presence of the LSB close to the pressure side trailing edge modifies the flow near the trailing edge and results in an increased camber as discussed in [18]. The pressure side LSB is then responsible for the increased slope of the $C_{\mathrm{L}}$ for $\mathrm{AoA} \in\left[2^{\circ} ; 5^{\circ}\right]$ and its sharp decrease between $\mathrm{AoA}=5^{\circ}$ and $\mathrm{AoA}=6.5^{\circ}$ contributes to the lift plateau. $C_{\mathrm{p}}$ distributions right before and right after the motion of the transition (Fig. 8) have different shapes with a pressure plateau located 


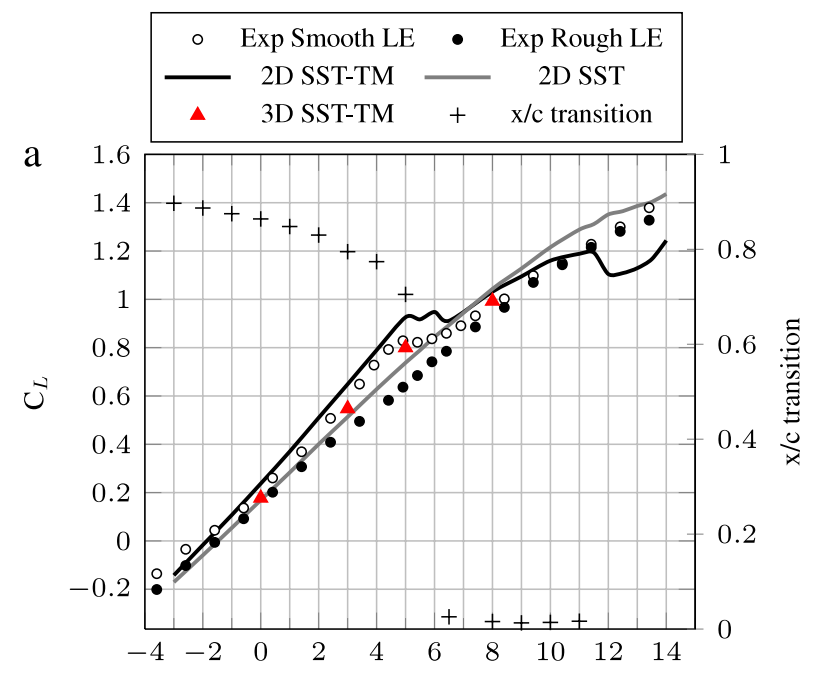

b
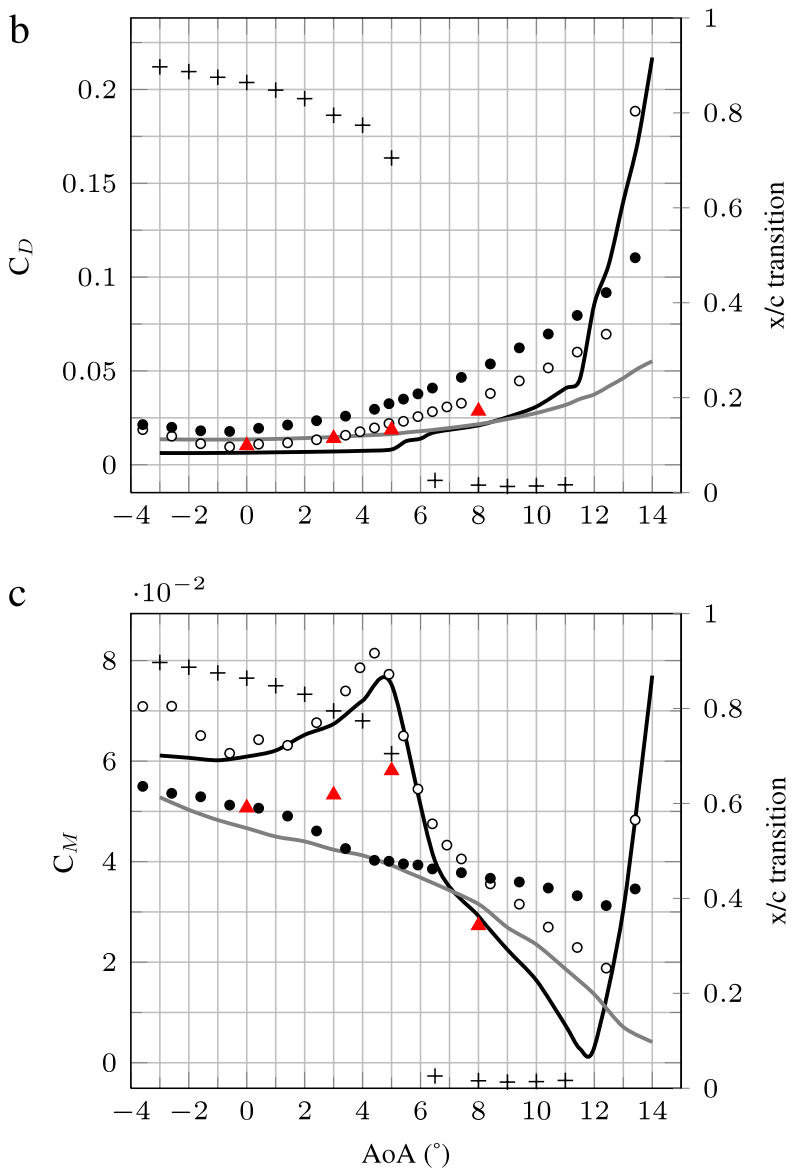

Fig. 7. Experimental vs. numerical lift (a), drag (b) and moment (c) coefficients, $\operatorname{Re}=7.5 \times 10^{5}$.

close to the trailing edge or close to the leading edge but lead to similar integrated values and then to similar $C_{\mathrm{L}}: C_{\mathrm{L}}\left(\mathrm{AoA}=5^{\circ}\right)=$ 0.926 and $C_{\mathrm{L}}\left(\mathrm{AoA}=6.5^{\circ}\right)=0.911$. Rough leading edge hydrofoil $C_{\mathrm{L}}$ starts from a lower value at $\mathrm{AoA}=-4^{\circ}$ than the smooth one and has a quasi-linear evolution at low angles of attack while the slope slightly decreases at high incidences. This behavior indicates that the added roughness has successfully triggered transition at the leading edge (no plateau). Smooth and rough leading edge hydrofoils' data are very similar from $\mathrm{AoA}=8^{\circ}$ to $\mathrm{AoA}=14^{\circ} .2 \mathrm{D}$ calculations present the same trends as experiments from $A o A=-4^{\circ}$ to $\mathrm{AoA}=9^{\circ}$. Beyond AoA $=9^{\circ}, C_{\mathrm{L}}$ predicted by SST-TM is lower than the SST value and starts oscillating at $11.5^{\circ}\left(1.5^{\circ}\right.$ earlier than

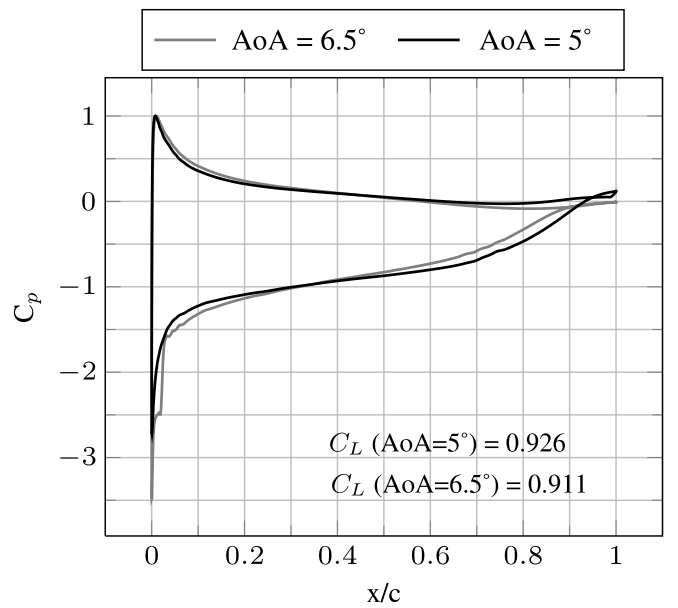

Fig. 8. SST-TM pressure coefficient distribution at $A o A=5^{\circ}$ and $A o A=6.5^{\circ}$, $\operatorname{Re}=7.5 \times 10^{5}$

experiments, with a higher amplitude). Oscillating $C_{\mathrm{L}}$ have been averaged to be displayed in Fig. 7(a). Numerical transition locations displayed in Fig. 7 show that the sudden motion of the transition point toward the leading edge corresponds to the lift plateau.

\subsubsection{Drag coefficient}

Figs. 7(b) and 9 (zoom of Fig. 7(b)) show measured and calculated drag coefficients. Rough and smooth leading edges $C_{D}$ show the same trends but rough leading edge values are significantly higher than smooth leading edge ones for all angles of attack except AoA $=13.5^{\circ}$. Drag coefficients of the rough leading edge hydrofoil were expected to be higher than those of the smooth hydrofoil because of the increased drag of the turbulent boundary layer but the significant gap between the two measurements also comes from the thickness and roughness of the sand paper added on the leading edge. Smooth leading edge $C_{D}$ increases sharply to a value close to 0.2 at $\mathrm{AoA}=13.5^{\circ}$. SST-TM calculations show that $C_{\mathrm{D}}$ increases very slowly from $-2^{\circ}$ to $5^{\circ}$ and then increases strongly to reach turbulent values at $A o A=6.5^{\circ}$ (Fig. 9). This step is not seen in the experiment. SST-TM $C_{D}$ starts increasing at AoA $=11.5^{\circ}, 1^{\circ}$ earlier than in the experiment. The $C_{\mathrm{D}}$ obtained with the SST calculation follows the same trend as the rough leading edge. 2D calculations show similar variations to experiments but there is an additional offset.

\subsubsection{Moment coefficient}

Fig. 7(c) shows measured and calculated moment coefficients. The positive values indicate that the foil tends to pitch nose down. Measurements show great differences between rough and smooth leading edge hydrofoils from AoA $=-4^{\circ}$ to AoA $=7^{\circ}$. Smooth leading edge $C_{\mathrm{M}}$ first decreases until $\mathrm{AoA}=-0.5^{\circ}$ but then reaches a peak at $\mathrm{AoA}=4.5^{\circ}$ before decreasing to rough-leading-edgelike values at $A o A=7.5^{\circ}$. Smooth leading edge $C_{M}$ is then lower than rough leading edge one from $8^{\circ}$ to $12^{\circ}$. $C_{\mathrm{M}}$ increases sharply at $\mathrm{AoA}=13 \cdot 5^{\circ}$. $2 \mathrm{D}$ calculations fit well with experiments except two main points: SST curve shows a modification of the slope at AoA $=6.5^{\circ}$ while the experiment shows a quasi-linear evolution and calculations start predicting very similar results at $\mathrm{AoA}=7^{\circ}$ against $\mathrm{AoA}=8.5^{\circ}$ in the experiments. Experimental values and 2D calculations do not present an offset on $C_{\mathrm{M}}$.

\subsection{4. $3 D$ calculations}

An offset exists on both lift and drag coefficients between experimental and numerical data and the authors wondered whether it could come from the 2D assumption. 3D calculations have then been run at $A \circ A=0^{\circ}, 3^{\circ}$ and $5^{\circ}$ with the SST-TM model while a calculation at AoA $=8^{\circ}$ only used the SST model since both SST 


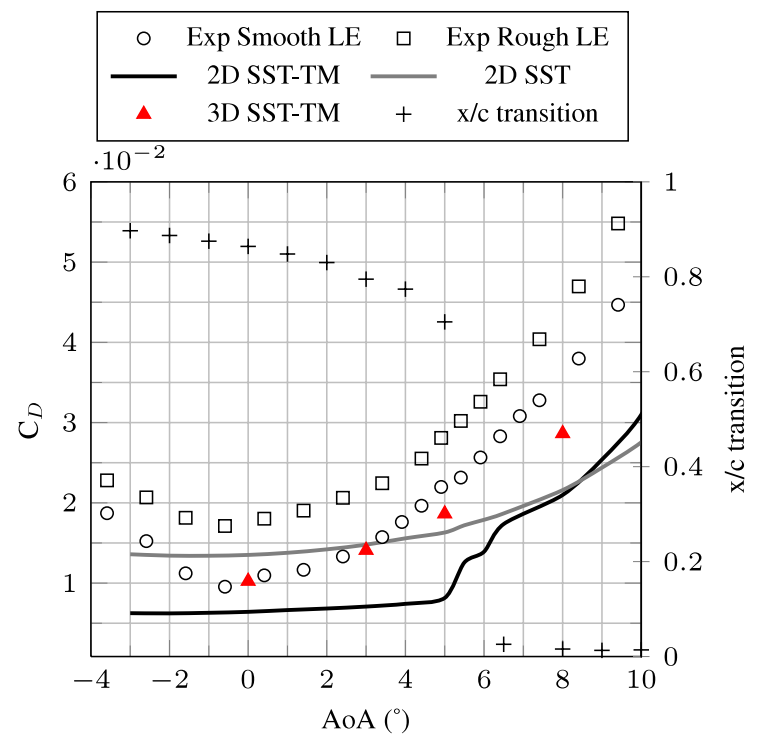

Fig. 9. Experimental vs numerical drag coefficients, zoom of Fig. 7(b), Re $=7.5 \times$ $10^{5}$.

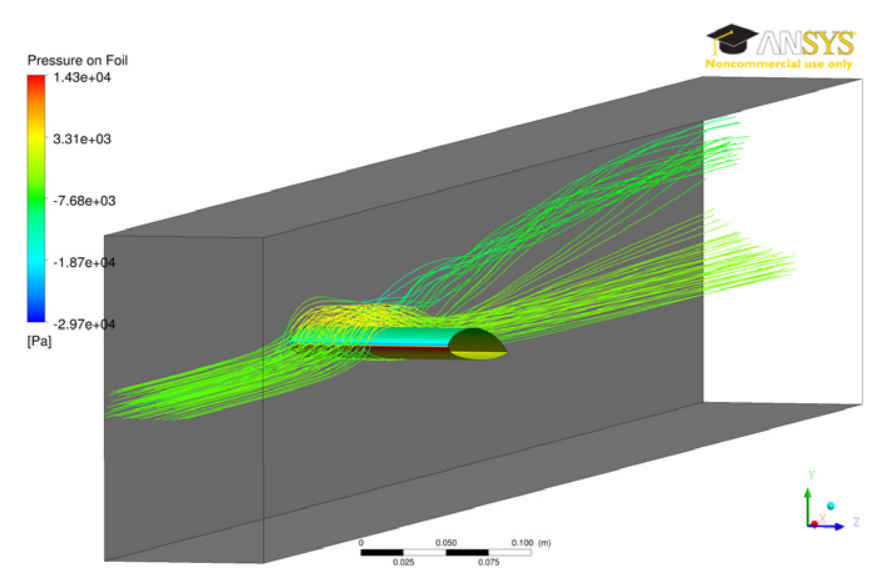

Fig. 10. Streamlines of the $3 D$ flow at $A o A=5^{\circ}$ Re $=7.5 \times 10^{5}$. (For interpretation of the references to color in this figure legend, the reader is referred to the web version of this article.)

and SST-TM models give very similar results in 2D calculations at this angle of attack. In the experimental test section, the distance between the foil and the side wall is very small so that we can assume that the foil is connected to the side wall. The boundary layer developing on the side wall encounters the foil and an appendage-body junction flow is formed. The adverse pressure gradient imposed by the foil causes separation of the boundary layer upstream of the leading edge and an intense recirculation is generated. Streamwise legs of this vortex stretch around the foil in a horseshoe vortex with each leg having circulation of opposite signs. Devenport et al. [19] established that the horseshoe vortex is subjected to low-frequency oscillations which make the numerical simulation difficult and will require transient calculations. Levchenya et al. [11] and Apsley et al. [20] showed that the k- $\omega$ SST turbulence model was the most adapted twoequation RANS turbulence model to predict the horseshoe vortex.

Transient calculations were required to reach convergence of the oscillating $C_{\mathrm{L}}$ and $C_{\mathrm{D}}$ and a time step of $10^{-3} \mathrm{~s}$ was used. $C_{\mathrm{L}}$, $C_{\mathrm{D}}$ and $C_{\mathrm{M}}$ displayed in Fig. 7(a)-(c) (red triangles) are averaged on at least 3 periods. The horseshoe vortex (Fig. 10) modifies the pressure and the wall shear stress distributions over the foil surface which leads to lower $C_{\mathrm{L}}$ and higher $C_{\mathrm{D}}$ than $2 \mathrm{D}$ calculations.
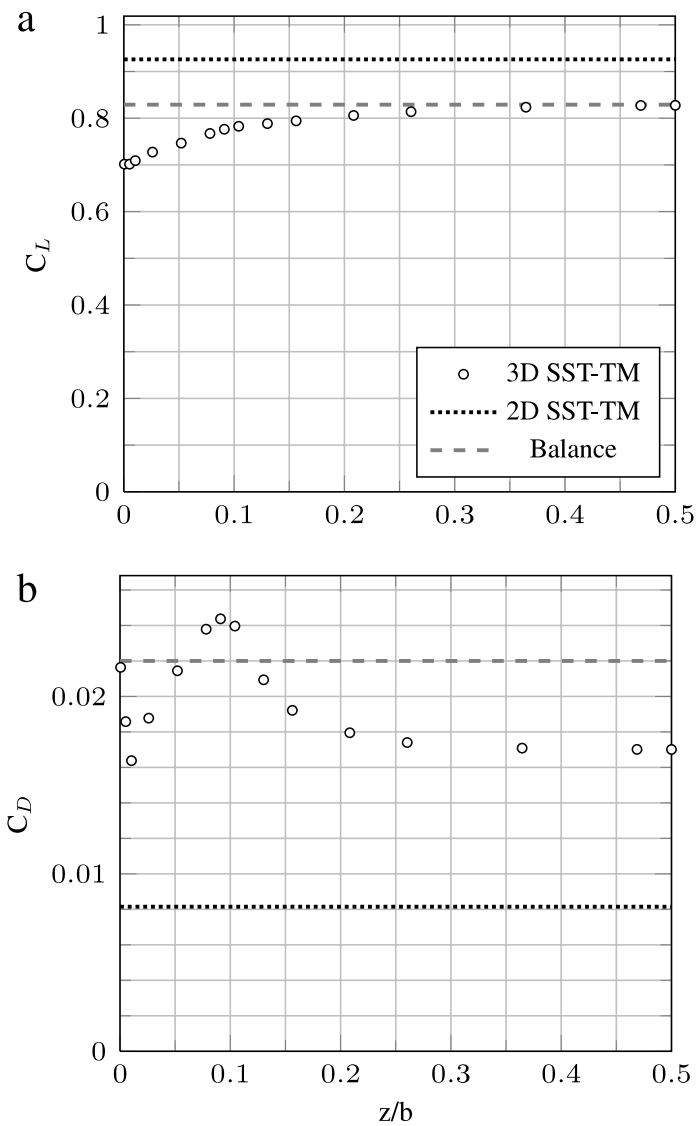

Fig. 11. Spanwise distribution of lift (a) and drag (b) coefficients from the 3D calculation plus 2D SST-TM and balance (experimental) averaged values, $\mathrm{AoA}=5^{\circ}$, $\operatorname{Re}=7.5 \times 10^{5} . b=0.192 \mathrm{~m}$ (total span of the hydrofoil).

Fig. 11 shows the spanwise distribution of $C_{\mathrm{L}}$ and $C_{\mathrm{D}}$ extracted from the $3 \mathrm{D}$ calculation at AoA $=5^{\circ}$. The influence of the side wall $(z / b=0)$ can be clearly seen on both $C_{\mathrm{L}}$ and $C_{\mathrm{D}}$ curves. The lift coefficient (Fig. 11(a)) starts from a low value close to the side wall and approaches the experimental value as an asymptote at mid span. The $3 \mathrm{D} C_{\mathrm{L}}$ calculation is close to the experimental data while the 2D SST-TM is 0.1 higher. The drag coefficient (Fig. 11(b)) first decreases close to the side wall, then increases and finally decreases to reach an asymptotic value at mid span. The 3D calculation is closer to the experimental value than the $2 \mathrm{D}$ calculation. It can be noticed that asymptotic values of $3 \mathrm{D} C_{\mathrm{L}}$ and $C_{\mathrm{D}}$ are not the $2 \mathrm{D}$ values. The $3 \mathrm{D}$ effect then generates a non homogeneous spanwise distribution of the coefficients but the horseshoe vortex also modifies the flow at mid span. $3 \mathrm{D} C_{\mathrm{L}}$ and $C_{\mathrm{D}}$ show very good agreement with experiments (Figs. 7(a) and (b) and 9). This confirms the 2D assumption is the reason of the offset observed between experiments and 2D calculations.

\subsection{Local validation, angle of attack $=2^{\circ}$}

This section aims at providing local validation to complete the global validation based on force coefficients. This way, velocity profiles in the vicinity of the LSB at AoA $=2^{\circ}$ are compared to experimental data from Ducoin et al. [21] (Fig. 12). SST-TM calculation shows very good agreement with the experimental data before and inside the LSB. The thickness of the LSB, at $x / c=0.79$, is particularly well predicted. The agreement decreases in the turbulent, reattached boundary layer $(x / c=0.9)$ but remains good, especially as compared to the SST calculation which shows, as expected, poor agreement with the experiment. 

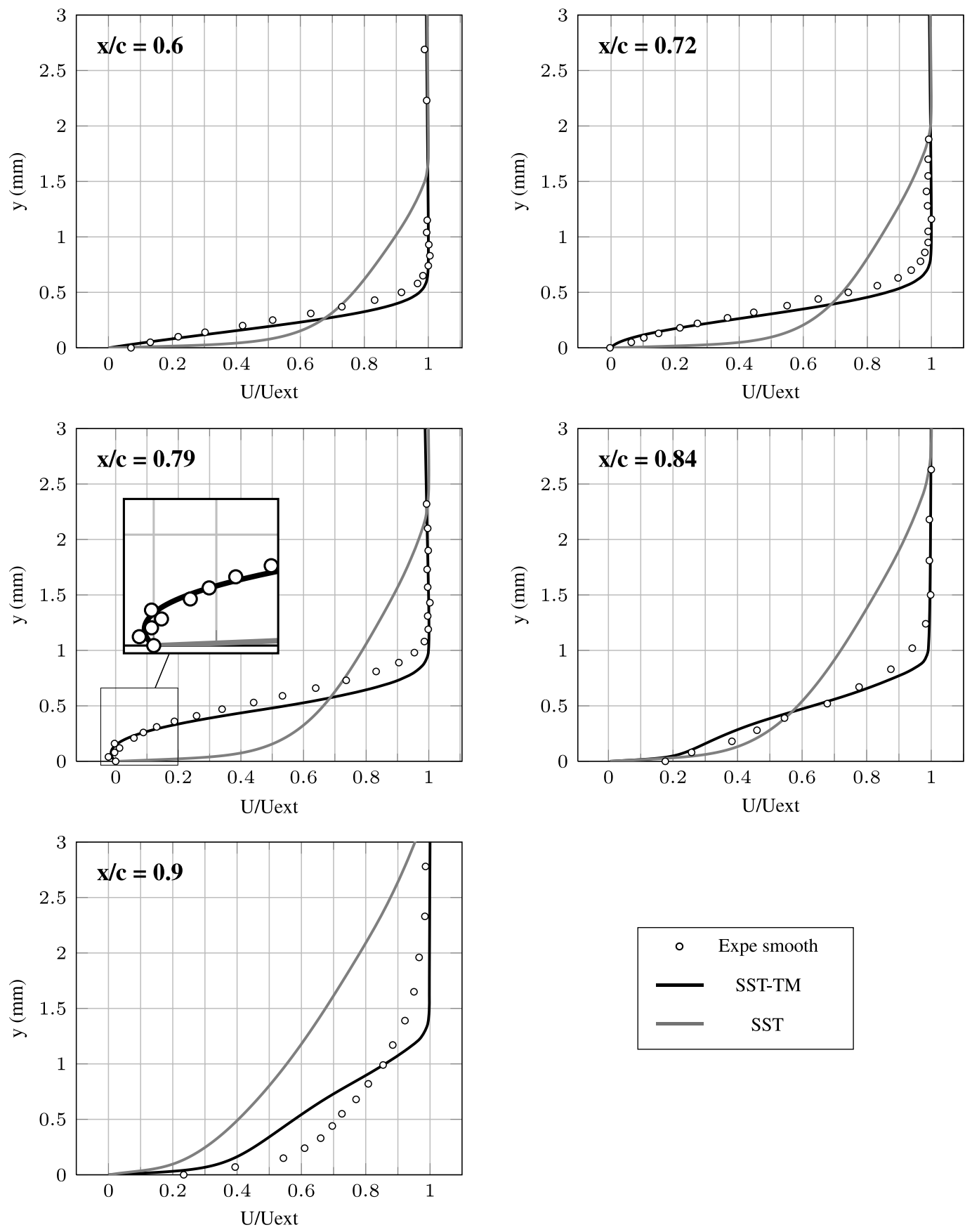

Fig. 12. Velocity profiles in the vicinity of the LSB at 5 chord locations, $A o A=2^{\circ}, \operatorname{Re}=7.5 \times 10^{5}$.

Fig. 13 displays the pressure coefficient distribution at $\mathrm{AoA}=$ $2^{\circ}$. SST-TM and SST data are compared to experimental data on the suction side. Experimental $C_{\mathrm{p}}$ are measured on a smooth hydrofoil with two different methods. Legend "Expe" of Fig. 13 refers to measurements presented in [6] using pressure transducers located at several chord locations of the suction side. Measurements are carried out on a pitching hydrofoil. Results of the quasi-static pitching motion are used in this paper to get the pressure coefficients at AoA $=2^{\circ}$. Legend "LDV" refers to velocity profiles presented in [21] using the Laser Doppler Velocimetry technique (also displayed in Fig. 12). Pressure coefficients are deduced from the velocity profiles according to Eq. (7).

$C_{\mathrm{p}}=1-\left(\frac{U_{\text {ext }}}{U_{\infty}}\right)^{2}$
Pressure transducers show good agreement with the SST-TM calculation from $x / c=0.3$ to $x / c=0.9$. The transducers located at $x / c=0.1$ and $x / c=0.2$ give $C_{\mathrm{p}}$ which are closer to the SST prediction than the SST-TM prediction. Pressure coefficients deduced from the velocity profiles (LDV) in the vicinity of the LSB show good agreement with the SST-TM calculation though there is a slight gap at the location of the LSB. The location of the pressure plateau is in very good agreement with the SST-TM calculation. Experimental $C_{\mathrm{p}}$ show better agreement with SST-TM than with SST.

Comparisons between numerical and experimental velocity profiles and pressure coefficients at AoA $=2^{\circ}$ show that the transition model gives accurate results whereas the fully turbulent model fails to predict the laminar and the transitional parts of the boundary layer. 


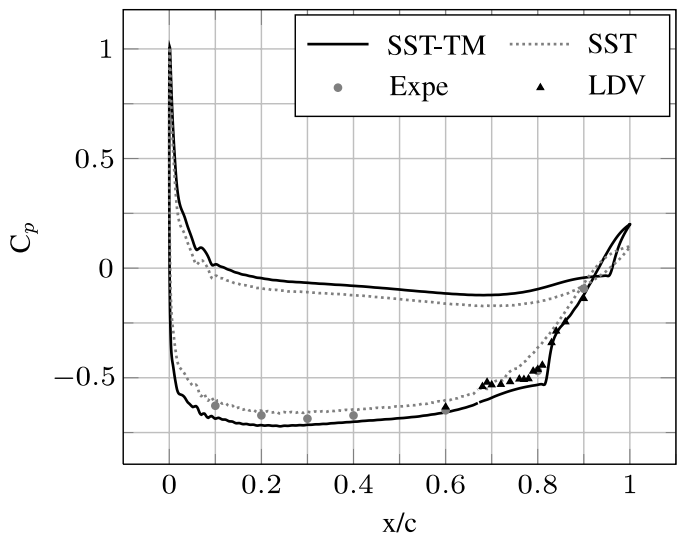

Fig. 13. SST-TM and SST pressure coefficient distributions compared to experimental $C_{p}$ from [6] (Expe) and [21] (LDV), AoA $=2^{\circ}, \operatorname{Re}=7.5 \times 10^{5}$

\subsection{Local analysis}

Section 5.1 showed that SST-TM and SST models behave well before $6.5^{\circ}$ with predictions very close to experimental data. Then both models predict similar values of $C_{\mathrm{L}}$ or $C_{\mathrm{D}}$ until $9^{\circ}$. Above $9^{\circ}$, a gap appears between both models' predictions while it does not exist in the experiments. This section aims at explaining these points by investigating local $C_{\mathrm{p}}$ and $C_{\mathrm{f}}$ distributions on the hydrofoil. Experimental $C_{\mathrm{p}}$ of Ducoin et al. [6] are compared to the calculations and show good agreement with the results of the SST-TM model (Fig. 14).

\subsubsection{Angle of attack $=3^{\circ}$}

At an angle of attack of $3^{\circ}$, SST model predicts a $C_{\mathrm{L}} 20 \%$ lower than the SST-TM model. This difference can be clearly seen on the $C_{\mathrm{p}}$ distribution (Fig. 14(a)) where a quasi constant $\Delta C_{\mathrm{p}}=$

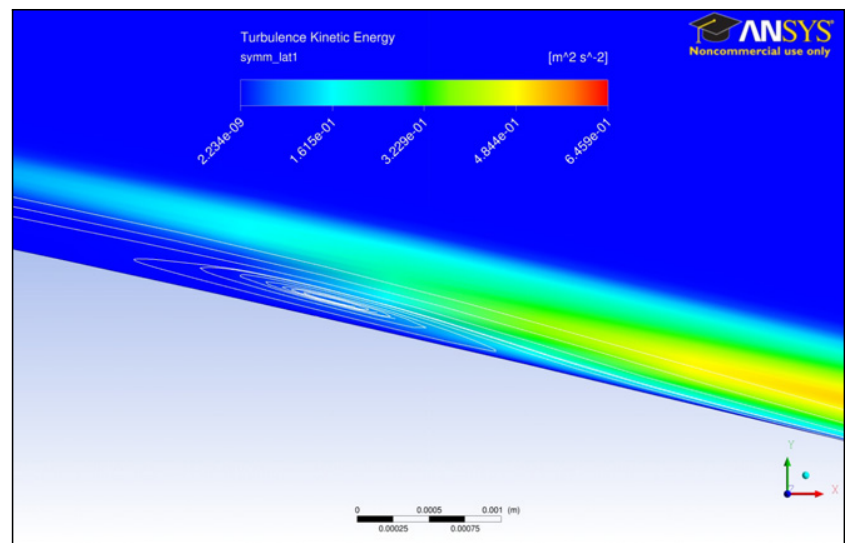

Fig. 15. Turbulent kinetic energy contours and streamlines in the vicinity of the $\operatorname{LSB}(x / c=0.78)$. AoA $=3^{\circ}, \operatorname{Re}=7.5 \times 10^{5}$. (For interpretation of the references to color in this figure legend, the reader is referred to the web version of this article.)

$\left|C_{\mathrm{pSST}-\mathrm{TM}}-C_{\mathrm{pSST}}\right|$ exists between the two models along the chord : $\Delta C_{\mathrm{p} \text { pressure side }}=0.045$ and $\Delta C_{\mathrm{p} \text { suction side }}=0.075 . \Delta C_{\mathrm{p}}$ comes from the difference of boundary layer states. SST-TM predicts a laminar boundary layer on the pressure side and on $80 \%$ of the suction side while SST predicts fully turbulent boundary layers. Laminar boundary layers generate lower $C_{\mathrm{f}}$ than turbulent ones which explains the difference between the two curves on Fig. 14(d). The LSB induced transition on the suction side occurs at $x / c=0.8$ where the $C_{\mathrm{f}}$ first falls to a trough before reaching turbulent levels. The LSB also induces a plateau on the $C_{\mathrm{p}}$ curve at $x / c=0.8$ (Fig. 14(a)). Fig. 15 presents a close view of the suction side LSB predicted by the SST-TM calculation. Contours of turbulent kinetic energy show that its production really starts at the end of the LSB which leads to transition. Transition on the pressure side occurs at the trailing edge $(x / c=0.98)$.
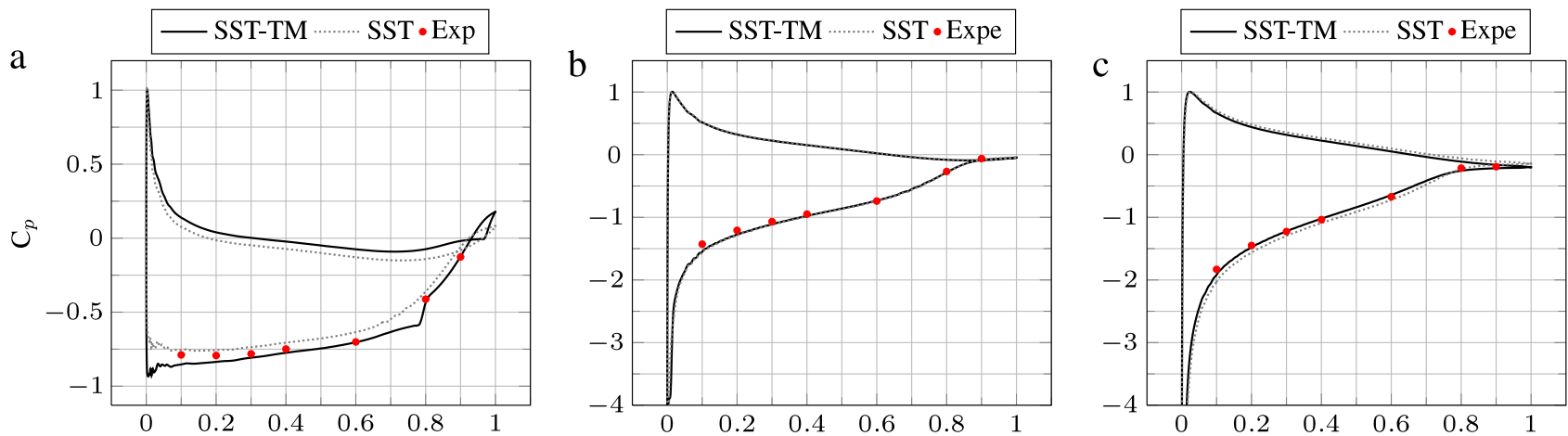

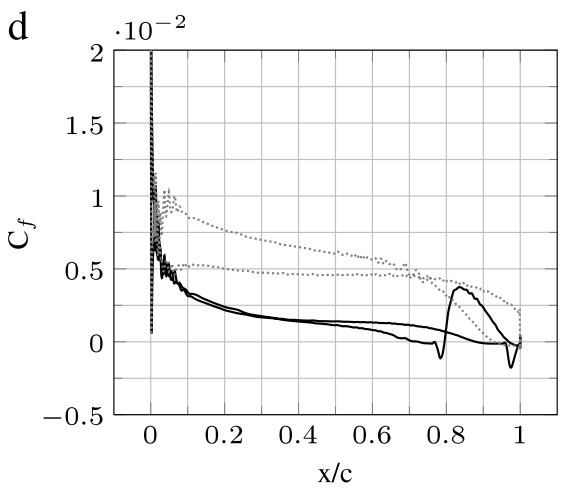

$\mathrm{AoA}=3^{\circ}$

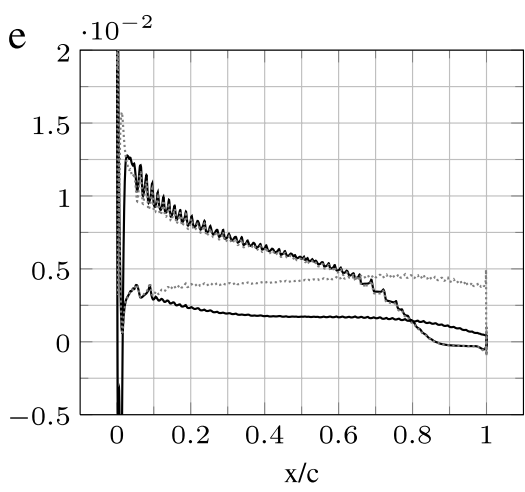

$\mathrm{AoA}=8^{\circ}$

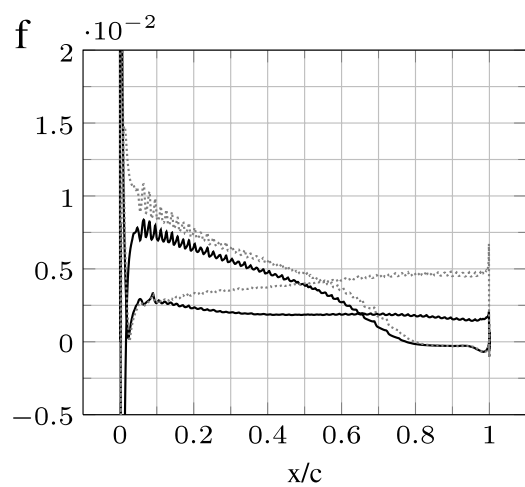

$\mathrm{AoA}=11^{\circ}$

Fig. 14. Pressure and friction coefficients at $A o A=3^{\circ}((\mathrm{a})$ and $(\mathrm{d})), 8^{\circ}((\mathrm{b})$ and $(\mathrm{e}))$ and $11^{\circ}((\mathrm{c})$ and $(\mathrm{f})), \operatorname{Re}=7.5 \times 10^{5}$. 


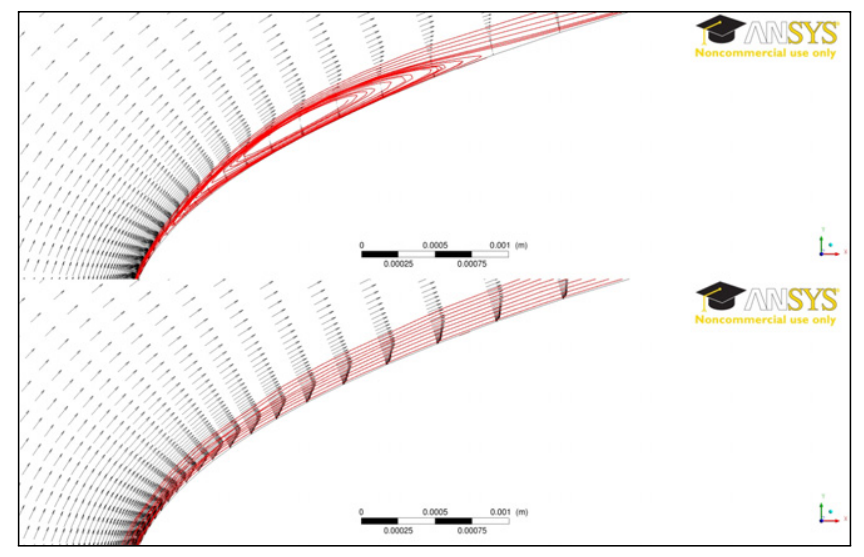

Fig. 16. Velocity vectors and streamlines in the vicinity of the LSB for both SST-TM (top) and SST (bottom) models. AoA $=11^{\circ}, \mathrm{Re}=7.5 \times 10^{5}$.

\subsubsection{Angle of attack $=8^{\circ}$}

At an angle of attack of $8^{\circ}$, SST and SST-TM predictions of $C_{\mathrm{L}}$ or $C_{D}$ are very similar. Fig. 14 (b) shows that $C_{p}$ distributions are nearly the same for both models even if a little plateau caused by the LSB can be seen close to $x / c=0$ (transition occurs at $x / c=0.016) . C_{\mathrm{p}}$ distributions are in very good agreement with experimental data of Ducoin et al. [6]. $\Delta C_{\mathrm{p}}$ are low : $\Delta C_{\mathrm{p} \text { pressure side }}=5.3 \times 10^{-3}$ and $\Delta C_{\mathrm{p} \text { suction side }}=7.5 \times 10^{-3}$ that is to say about a tenth of the $\Delta C_{\mathrm{p}}$ obtained at $3^{\circ}$. This behavior is due to the presence of the LSB at the leading edge which induces transition of the boundary layer. Both SST and SST-TM models then predict a turbulent boundary layer on the suction side. This is confirmed by the $C_{\mathrm{f}}$ distribution (Fig. 14(e)) which is the same for both models on the suction side, except the trough at the leading edge due to the LSB. Both models however predict slightly different $C_{\mathrm{p} \text { min }}$ values : $C_{\mathrm{p} \text { min }}=-5.53$ with SST while $C_{\mathrm{p} \text { min }}=-5.16$ with SST-TM. Boundary layer on the pressure side is laminar with SST-TM model according to the low levels of $C_{\mathrm{f}}$ as compared to the SST ones. However, laminar and turbulent boundary layers lead to very similar $C_{p}$ distributions on the pressure side at this angle of attack.

\subsubsection{Angle of attack $=11^{\circ}$}

For AoA $=11^{\circ}$, significant gaps appear between SST and SST-TM predictions of $C_{\mathrm{L}}$ and $C_{\mathrm{D}}$. The SST-TM calculation predicts a lower $C_{\mathrm{L}}$ than the SST one (Fig. 7). Fig. 14(c) shows that $C_{\mathrm{p}}$ curves are no longer superimposed: SST-TM calculation leads to the inner curve, in agreement with the experimental data, and then to a lower $C_{\mathrm{L}} . \Delta C_{\mathrm{p}}$ between the two curves are quasi constant along the chord and of the same order as the $3^{\circ}$ case: $\Delta C_{\mathrm{p} \text { suction side }}=0.081$ and $\Delta C_{\text {p pressure side }}=0.034$. Transition occurs at $x / c=0.016$, as for $A o A=8^{\circ}$. The difference of $C_{p \text { min }}$ is significant between both predictions: $C_{\mathrm{p} \text { min }}=-10.26$ with SST while $C_{\mathrm{p} \text { min }}=-8.07$ with SST-TM. Like the $8^{\circ}$ case, SST calculation predicts a lower $C_{\mathrm{p} \text { min }}$ than SST-TM at AoA $=11^{\circ}$. This behavior and the gap between experimental and SST-TM data at high incidences tend to point out a limitation of the $\gamma-\operatorname{Re}_{\theta}$ Transition Model.

It should be noted that the SST calculation predicts a small vortex at the leading edge for AoA $=11^{\circ}$ but its size is very small: $0.2 \%$ of the chord against $1.4 \%$ of the chord for the LSB of the SST-TM calculation (Fig. 16).

\subsection{Comparative results}

Sections 5.1 and 5.3 have mentioned differences between SST and SST-TM predictions that should be of interest for the design of lifting bodies. These differences are related to physics until AoA $=9^{\circ}$ while some doubts remain in the reliability of the
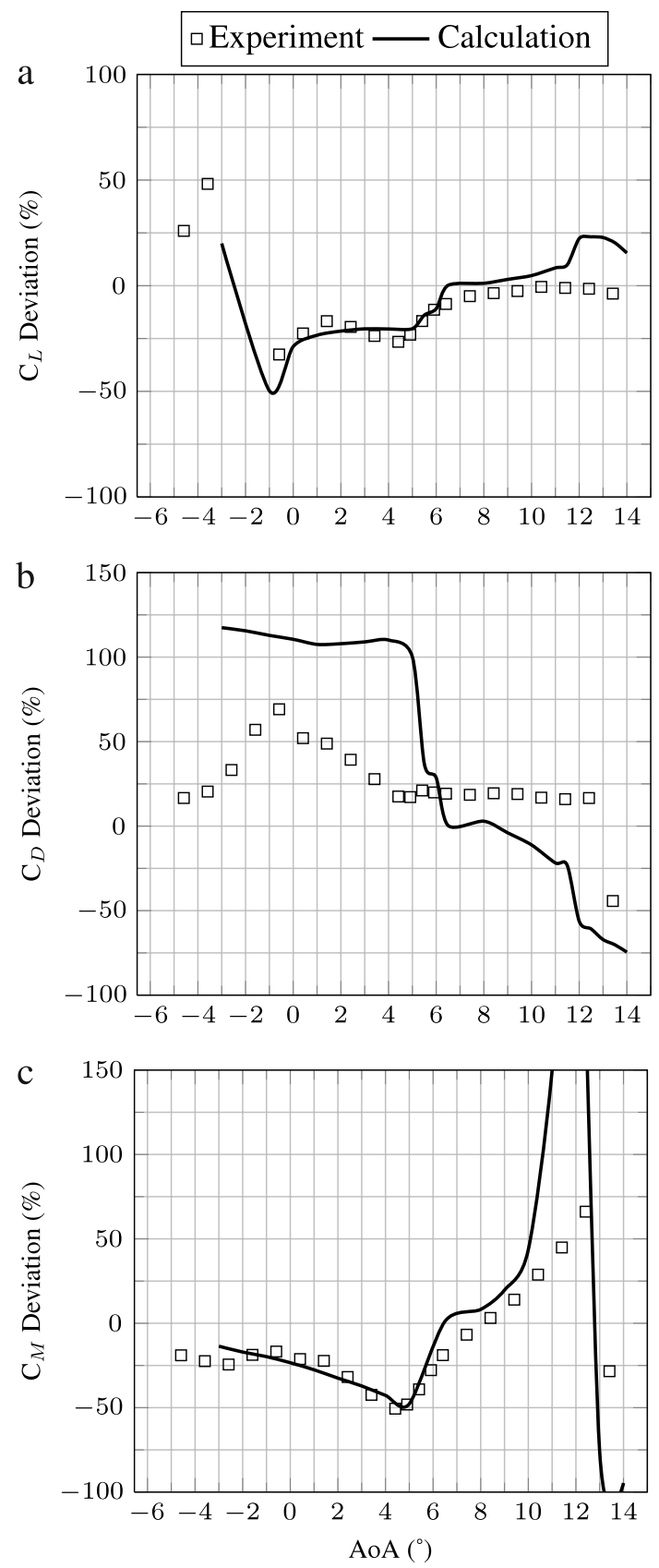

Fig. 17. Deviations of experimental and numerical fully turbulent values of $C_{\mathrm{L}}$ (a), $C_{\mathrm{D}}$ (b) and $C_{\mathrm{M}}(\mathrm{c})$ relative to transitional values, $\mathrm{Re}=7.5 \times 10^{5}$.

model for higher incidences, on this hydrofoil. To help choose between SST and SST-TM models, Fig. 17 displays the gaps in percentage between both models predictions of $C_{\mathrm{L}}, C_{\mathrm{D}}$ and $C_{\mathrm{M}}$ coefficients with SST-TM results taken as the reference (e.g. $\left.C_{\mathrm{L} \text { Deviation }}=\left(C_{\mathrm{L} \text { SST }}-C_{\mathrm{L} \text { SST-TM }}\right) / C_{\mathrm{L} \text { SST-TM }} \times 100\right)$. Corresponding deviations of experimental $C_{\mathrm{L}}, C_{\mathrm{D}}$ and $C_{\mathrm{M}}$ are plotted in Fig. 17 with smooth leading edge hydrofoil data taken as the reference. Experimental data fit correctly with 2D computational results:

- from $0^{\circ}$ to $6^{\circ}$, SST calculations under predict $C_{\mathrm{L}}(-20 \%)$ and over predict $C_{\mathrm{D}}(+110 \%)$

- from $6^{\circ}$ to $9^{\circ}$, both SST and SST-TM calculations lead to the same predictions

- from $9^{\circ}$ to $14^{\circ}$, SST and SST-TM predictions differ which is not observed on experimental data.

Modeling the laminar to turbulent transition is then very interesting if the lifting body operates at low angles of attack or more 
precisely if it operates at angles of attack for which the transition is located close to the trailing edge. However if it operates at angles of attack for which the transition is located close to the leading edge, a fully turbulent simulation provides accurate results and is more efficient. Beyond $9^{\circ}$, the SST-TM calculations predict $C_{\mathrm{L}}$ that differ from experiments but they have the advantage of predicting a sharp increase of $C_{D}$ earlier than the SST ones, which is in agreement with the experiments and can be relevant for design issues.

\section{Conclusion}

The SST $\gamma-\operatorname{Re}_{\theta}$ transition model implemented in the CFX ${ }^{\circledR}$ flow solver has been used to study the effect of the laminar separation bubble induced transition on the lift, drag and moment coefficients of a NACA66 hydrofoil. Angles of attack ranged from $-4^{\circ}$ to $14^{\circ}$ and the Reynolds number was $\operatorname{Re}=7.5 \times 10^{5}$. Verification was conducted with four different grids and four different time steps to get fully converged results. Experiments measuring $C_{\mathrm{L}}, C_{\mathrm{D}}$ and $C_{\mathrm{M}}$ with a 3-component hydrodynamic balance were also carried out in the IRENav's hydrodynamic tunnel. Two sets of experiments were conducted. Experiments on a smooth hydrofoil enabled comparison with RANS calculations using the transition model. Experiments on a hydrofoil with a roughness (sandpaper) added on the leading edge enabled comparison with RANS calculations using the SST turbulence model. The trends of lift, drag and moment coefficients were well predicted by 2D calculations. The accuracy of the coefficients, compared to experiments, was improved with 3D calculations. When comparing SST and SST-TM calculations, SST predicted a lower $C_{\mathrm{L}}(-20 \%)$ and a $C_{\mathrm{D}}$ twice as high as the SST-TM one, for angles of attack ranging from $0^{\circ}$ to $6^{\circ}$. From $6^{\circ}$ to $9^{\circ}$, predictions of both models were the same since the boundary layer of the suction side is turbulent in both SST and SST-TM calculations. Beyond $9^{\circ}$, SST-TM predictions diverged from SST ones which is not in agreement with the experiments. Velocity profiles in the vicinity of the LSB at an angle of attack of $2^{\circ}$ were extracted from the SST-TM calculation and were compared with a published experimental study. Numerical (SST-TM) and experimental velocity profiles showed very good agreement. Pressure and friction coefficients were studied at angles of attack $3^{\circ}, 8^{\circ}$ and $11^{\circ}$ to show the influence of the laminar separation bubble induced transition on lift, drag and moment coefficients. Pressure coefficients obtained from a published experimental study showed good agreement with the SST-TM results. The SST $\gamma-\operatorname{Re}_{\theta}$ transition model proved to be a relevant prediction tool for lifting bodies with smooth surfaces operating at a moderate Reynolds number.

\section{Acknowledgment}

The authors thank the technical staff of IRENav for their contribution to the experimental set up.

\section{References}

[1] B. Abu-Ghannam, R. Shaw, Natural transition of boundary layers - the effects of turbulence, pressure gradient, and flow history, J. Mech. Eng. Sci. 22 (5) (1980) 213-228.

[2] D. Wilcox, Simulation of transition with a two-equation turbulence model AIAA J. 32 (2) (1994) 247-255.

[3] D.D. Pasquale, A. Rona, S.J. Garrett, A selective review of CFD transition models, in: AIAA Fluid Dynamics Conference, 2009.

[4] F. Menter, R. Langtry, S. Volker, Transition modelling for general purpose CFD codes, Flow Turbul. Combust. 77 (2006) 277-303.

[5] J.N.N. Counsil, K. Goni Boulama, Validating the URANS shear stress transport $\gamma-\operatorname{Re} \theta$ model for low-Reynolds-number external aerodynamics, Int. J. Numer. Methods Fluids 69 (8) (2012) 1411-1432.

[6] A. Ducoin, J. Astolfi, F. Deniset, J. Sigrist, Computational and experimental investigation of flow over a transient pitching hydrofoil, Eur. J. Mech. B Fluids 28 (2009) 728-743.

[7] R. Lanzafame, S. Mauro, M. Messina, Wind turbine CFD modeling using a correlation-based transitional model, Renew. Energy 52 (2013) 31-39.

[8] D.K. Walters, D. Cokljat, A three-equation Eddy-viscosity model for Reynoldsaveraged Navier-Stokes simulations of transitional flow, J. Fluid Eng. 130 (2008) 1-14.

[9] M.S. Genç, Ü Kaynak, H. Yapici, Performance of transition model for predicting low aerofoil flows without/with single and simultaneous blowing and suction, Eur. J. Mech. B Fluids 30 (2) (2011) 218-235.

[10] ANSYS ICEM CFD User Manual, vol. 12.1, ANSYS, 2009

[11] A. Levchenya, E. Smirnov, V. Goryachev, RANS-based numerical simulation and visualization of the horseshoe vortex system in the leading edge endwall region of a symmetric body, Int. J. Heat Fluid Flow 31 (2010) 1107-1112.

[12] F. Menter, Two-equation Eddy-viscosity turbulence models for engineering applications, AIAA J. 32 (8) (1994) 1598-1605.

[13] F.R. Menter, M. Kuntz, R. Langtry, Ten years of industrial experience with the SST turbulence model, in: K.N.Y. Hankjalic, M. Tummers (Eds.), in: Turbulence, Heat and Mass Transfer, vol. 4, 2003.

[14] J. Ferziger, M. Peric, Computational Methods for Fluid Dynamics, Springer, New York, 2002.

[15] ANSYS CFX Solver Modeling Guide, Vol. 12.1, ANSYS, 2009

[16] M. Alam, N.D. Sandham, Direct numerical simulation of laminar separation bubbles with turbulent reattachment, J. Fluid Mech. 410 (2000) 1-28.

[17] R. Hain, C.J. Kähler, R. Radespiel, Dynamics of laminar separation bubbles at low-Reynolds-number aerofoils, J. Fluid Mech. 630 (2009) 129-153.

[18] W. Timmer, Two-dimensional low-Reynolds number wind tunnel results for airfoil NACA 0018, Wind Eng. 32 (6) (2008) 525-537.

[19] W.J. Devenport, R.L. Simpson, Time-dependent and time-averaged turbulence structure near the nose of a wing-body junction, J. Fluid Mech. $210(1990)$ 23-55.

[20] D. Apsley, M. Leschziner, Investigation of advanced turbulence models for the flow in a generic wing-body junction, Flow Turbul. Combust. 67 (1) (2001) 25-55.

[21] A. Ducoin, J. Astolfi, M. Gobert, An experimental study of boundary-layer transition induced vibrations on a hydrofoil, J. Fluids Struct. 32 (0) (2012) 37-51. 